\title{
Sistema de interação computacional baseado na detecção das mãos utilizando classificadores em cascata
}

\author{
Anne Livia da F. Macedo ${ }^{1}$, Igor Ruiz Gomes ${ }^{1}$ \\ ${ }^{1}$ Faculdade de Computação - Universidade Federal do Pará (UFPA) \\ Castanhal - PA - Brazil \\ \{annelivia16, ruiz.igor\}@gmail.com
}

\begin{abstract}
Resumo. Com o avanço da tecnologia, sistemas que possibilitam uma interação humano-computador mais natural e acessível, terão um impacto muito positivo na sociedade. Diante disso, esta pesquisa propõe o desenvolvimento de um processo de software de baixo custo computacional que permite a interação a distância com o computador através da detecção das mãos via webcam, utilizando o algoritmo de classificação Haar Cascade. Os resultados obtidos foram considerados satisfatórios ao indicar uma precisão de $82 \%$, recall de $80 \%$, F1-score de $81 \%$ e um tempo de resposta adequado e eficiente para a interação com diversas aplicações computacionais em tempo real.
\end{abstract}

\section{Introdução}

Em conformidade com [Oudah et al. 2020], os gestos com as mãos são uma das formas de comunicação não verbal que podem ser usadas em diversos campos, como controle de robôs e interação humano-computador (IHC). Para [Sun et al. 2020], a simplificação do processo de interação humano-computador tornou-se um ponto de pesquisa muito relevante quando se trata de sistemas de controle inteligente. Segundo [Cardoso et al. 2015], as soluções baseadas em gestos com as mãos mais eficazes fazem uso de dispositivos eletromecânicos como luvas ou sensores. Contudo, esses equipamentos são normalmente muito custosos, exigem procedimentos de calibração complexos, e não fornecem uma interface totalmente intuitiva.

Com o avanço expressivo da tecnologia da informação nos últimos anos, os esforços para o desenvolvimento de sistemas que permitam uma interação com as máquinas de maneira mais natural e acessível, terão um impacto muito positivo para a população, principalmente no que tange a acessibilidade digital. Diante desse contexto e almejando contribuir com pesquisas na área, este trabalho propõe o desenvolvimento de um sistema de baixo custo computacional que, utilizando apenas a webcam do computador e algoritmos de visão computacional, viabiliza o controle das teclas direcionais através da detecção das mãos do usuário em tempo real.

\section{Metodologia}

A principal contribuição desta pesquisa consiste na implementação de um sistema de baixo custo computacional, prático e acessível, apto a permitir a interação a distância com o computador através, exclusivamente, da detecção das mãos (gesto estático), sem a necessidade do uso de hardwares e sensores externos, complexos, de alto custo e não acessíveis. 
Para concretizar a proposta do projeto, foi implementado um programa que permite que o usuário possa acionar as teclas direcionais através do posicionamento das mãos em determinadas regiões do vídeo que é capturado em tempo real pela webcam do computador, utilizando um algoritmo de aprendizagem de máquina supervisionado e algumas técnicas de processamento digital de imagens. O desenvolvimento foi composto de três etapas principais: (1) treinamento do algoritmo de classificação Haar Cascade; (2) aplicação do classificador treinado para a detecção das mãos; (3) definição das regiões responsáveis por representar cada uma das teclas de interesse (cima, baixo, esquerda e direita). O programa foi desenvolvido através da linguagem de programação $\mathrm{C}++$, com o suporte dos algoritmos pré-implementados da biblioteca OpenCV 4.1.0. Além disso, o treinamento do classificador foi realizado baseando-se no trabalho de [Rezaei 2014], que disponibiliza aplicações essenciais para gerar a cascata de classificadores Haar-Like.

\subsection{Treinamento do classificador Haar Cascade}

O Haar Cascade é um algoritmo para detecção de objetos que tem como fundamento a abordagem de aprendizagem de máquina proposta por [Viola e Jones 2001], em que uma função em cascata é treinada a partir de muitas imagens positivas e negativas, para posteriormente efetivar a detecção de objetos em outras imagens. Esta abordagem oferece técnicas inteligentes de baixo custo computacional que permite o treinamento para detecção de objetos em circunstâncias adversas como a baixa iluminação [Pereira 2017].

Para efetuar o treino do classificador são necessários um conjunto de amostras que exibe o alvo de interesse (imagens positivas) e um conjunto que não apresenta o alvo (imagens negativas). Portanto, para compor as amostras positivas, foram consideradas 2496 imagens contendo apenas as mãos fechadas com a palma voltada para a frente e o polegar ao lado do indicador, conforme a Fig. 1. Algumas imagens foram coletadas através da webcam utilizada no desenvolvimento e outras são oriundas da internet e das bases de dados Hand Gesture Database [Maqueda et al. 2015] e Significant (ASL) Sign Language Alphabet Dataset [Muvezwa 2019].
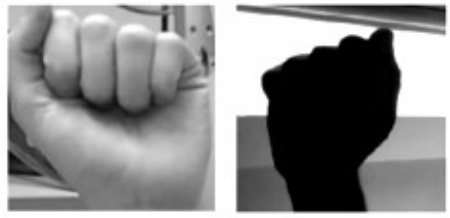
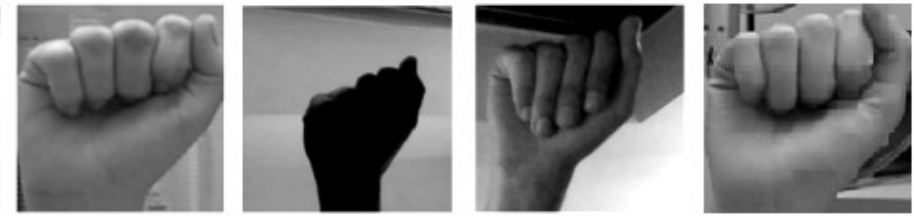

Figura 1. Amostras positivas.

Em relação ao conjunto de negativos, foram empregues 7994 imagens, na qual algumas eram aleatórias e outras apresentavam regiões faciais e do corpo humano para indicar um background tematicamente similar aos que são encontrados nas amostras positivas. Esses dados foram obtidos através da internet e do conjunto de imagens Real and fake face detection [Yonsei University 2019]. Após a elaboração das amostras, foi realizado o treinamento do classificador que durou aproximadamente 16 horas.

\subsection{Detecção das mãos}

A captura das mãos foi efetuada através do método detectMultiscale pertencente ao $O p e n C V$ que implementa a classificação em cascata percorrendo a imagem de entrada 
em diferentes escalas e retorna, após todos os procedimentos, uma lista de retângulos, indicando os locais onde as mãos foram encontradas com sucesso.

Inicialmente faz-se a conversão do frame obtido em tempo real para a escala de cinza e a equalização do histograma da imagem. Após o pré-processamento, foi especificado como argumentos para os parâmetros do método o valor 1.09 para o fator de escala, que determina o quanto uma imagem será redimensionada em cada uma das escalas. O valor 30 para a quantidade mínima de vizinhos, que objetiva evitar falsas detecções, fazendo com que neste caso, somente os objetos que apresentarem pelo menos 30 detecções sobrepostas sejam considerados, e por fim, foi estabelecido que somente os objetos com dimensões mínimas de 110x110 pixels, serão considerados.

\subsection{Interação com o computador}

A resolução da webcam integrada é de $640 \times 480$ pixels e optou-se pelo espelhamento da imagem de modo que o movimento em tempo real esteja na mesma direção que o movimento realizado pelo usuário. Em seguida, foi mapeada e determinada as regiões de interesse (ROIs) nos frames, de forma que cada uma das áreas indicadas represente uma tecla diferente. Os posicionamentos de cada ROI foram determinados estrategicamente com a intensão de aumentar a agilidade durante os acionamentos sucessivos e permitir uma representação simbólica do que cada área retangular corresponde. $\mathrm{O}$ algoritmo avalia qual das teclas deve ser acionada através da detecção da cor do retângulo obtido pelo método de classificação ao localizar as mãos. As coordenadas $\left(\mathrm{x}^{1}, \mathrm{y}^{1}\right)$ e $\left(\mathrm{x}^{2}, \mathrm{y}^{2}\right)$ das ROIs são: cima $(420,180)$ e $(500,250)$; baixo $(420$, $340)$ e $(500,410)$; esquerda $(320,260)$ e $(400,330)$; direita $(520,260)$ e $(600,330)$.

\section{Resultados}

Para avaliar os acionamentos sucessivos, foi desenvolvida uma aplicação que permite a movimentação do personagem para as quatro direções. Essa interface possui dimensões de 500x500 pixels e ao especificar uma orientação, o personagem terá um deslocamento de 20px para a direção indicada. A Fig. 2 exibe a interface da aplicação, o resultado da detecção das mãos em tempo real e o processo de acionamento das direções ao posicionar a mão sobre as ROIs referentes as teclas para a direita (a), cima (b), esquerda (c) e para baixo (d), respectivamente.

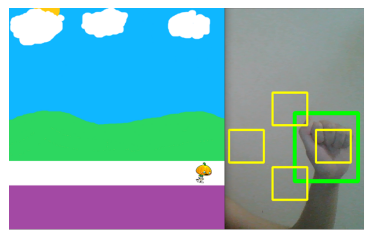

(a) Direita

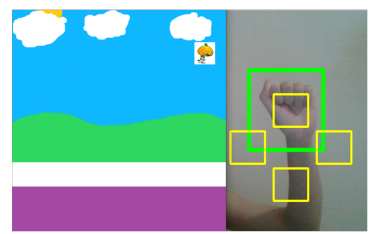

(b) Cima

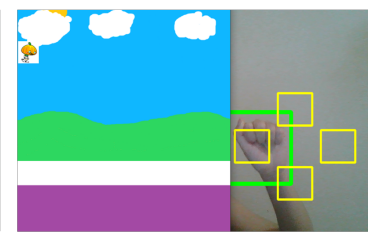

(c) Esquerda

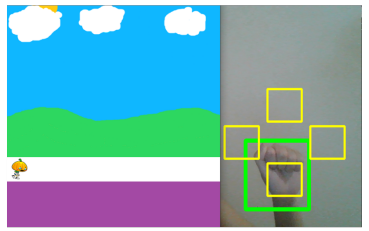

(d) Baixo

Figura 2. Processo de detecção das mãos em tempo real.

Em conformidade com a Fig. 2, para completar todo o percurso do personagem para a direita (a) e para a esquerda (c), são necessários 21 acionamentos cada. Já para cima (b) e para baixo (d) são requeridos 13 acionamentos cada. Baseando-se nesta quantidade de acionamentos das teclas direcionais (68 ao todo), foi apurado o tempo de resposta médio para completar todos os percursos do personagem seguindo a ordem de movimentação da Fig. 2. Três pessoas realizaram o experimento seis vezes e os tempos 
médios para o primeiro, segundo e terceiro participante, com precisão de $1 / 100 \mathrm{~s}$, foram de 5.91, 7.18 e 8.27 segundos, respectivamente. O desempenho do detector das mãos foi avaliado com 100 imagens do dataset de Maqueda et al. (2015). A Tab. 1 exibe a quantidade de verdadeiros positivos (VP), falsos positivos (FP) e falsos negativos (FN), assim como as taxas de precisão $(\mathrm{P})$, recall $(\mathrm{R})$ e F1-score $(\mathrm{F} 1)$.

Tabela 1. Desempenho do detector das mãos.

\begin{tabular}{cccccc}
\hline VP & FP & FN & P & R & F1 \\
\hline 80 & 18 & 20 & $82 \%$ & $80 \%$ & $81 \%$ \\
\hline
\end{tabular}

\section{Conclusão}

Esta pesquisa propôs o desenvolvimento de um sistema de baixo custo computacional que permite a interação a distância com o computador através da detecção das mãos em tempo real, utilizando um algoritmo de classificação em cascata baseado em características Haar. $\mathrm{O}$ modelo proposto alcançou resultados satisfatórios ao propiciar o acionamento das teclas direcionais em um tempo de resposta eficiente e adequado para a execução de inúmeras aplicações computacionais. Ademais, pôde-se concluir que o detector das mãos treinado obteve um desempenho geral apropriado quando considerado os valores obtidos ao computar as métricas de avaliação conhecidas como precisão, recall e F1-score. Para trabalhos futuros, pretende-se aprimorar a performance do sistema e incluir novas funcionalidades como o controle do cursor do mouse.

\section{Referências}

Cardoso, T., Delgado, J. and Barata, J. (2015). Hand gesture recognition towards enhancing accessibility. Procedia Computer Science, 67:419-429.

Maqueda A. I., et al. (2015). Human-computer interaction based on visual hand-gesture recognition using volumetric spatiograms of local binary patterns, Computer Vision and Image Understanding, Special Issue on Pose \& Gesture, 141:126-137.

Muvezwa, K. (2019). Significant (ASL) Sign Language Alphabet Dataset. Disponível em: https://www.kaggle.com/kuzivakwashe/significant-asl-sign-language-alphabet-dataset.

Acesso em: 31 ago. 2021.

Oudah, M., Al-Naji, A. and Chahl, J. (2020). Hand gesture recognition based on computer vision: a review of techniques. Journal of Imaging, 6(8):73.

Pereira, R. C. (2017). Técnica de rastreamento e perseguição de alvo utilizando o algoritmo Haar Cascade aplicada a robôs terrestres com restrições de movimento. Master's thesis, Universidade Federal do Rio Grande do Norte, Natal, Brasil.

Rezaei, M. (2014). Creating a cascade of haar like classifiers Step by step.

Sun, Y., et al. (2020). Intelligent human computer interaction based on non redundant EMG signal. Alexandria Engineering Journal, 59(3):1149-1157.

Viola, P. and Jones, M. (2001). Rapid object detection using a boosted cascade of simple features, In: Proceedings of the 2001 IEEE computer society conference on computer vision and pattern recognition - CVPR 2001.

Yonsei University, CIPLAB. (2019). Real and Fake Face detection. Disponível em: https://www.kaggle.com/ciplab/real-and-fake-face-detection. Acesso em: 31 ago. 2021. 